\title{
Retour d'expérience des attentats du 13 novembre 2015. Prise en charge médicale préhospitalière
}

\section{Feedback on Terrorist Attacks on November 13, 2015. Prehospital Medical Care}

\author{
B. Frattini $\cdot$ S. Boizat $\cdot$ S. Travers $\cdot$ M. Bignand $\cdot$ J.-P. Tourtier \\ Reçu le 11 janvier 2016; accepté le 13 janvier 2016 \\ (C) SFMU et Lavoisier SAS 2016
}

\begin{abstract}
Résumé Lors des attentats qui ont frappé Paris et SaintDenis le 13 novembre 2015, les secours médicaux préhospitaliers ont été confrontés à une situation inédite et complexe : la prise en charge de nombreuses victimes de plaies par balles, sur des sites multiples, dans un contexte de sécurité incertaine. La mise en œuvre du concept tactique du plan rouge alpha (extraction, triage, damage control, régulation et évacuation rapide) et la qualité des relations entre services de secours (exercices communs, bonne communication) ont permis de gérer au mieux cette situation de crise.
\end{abstract}

Mots clés Multivictimes · Triage · Prise en charge médicale - Damage control préhospitalier

\begin{abstract}
During the terrorist attacks that have hit Paris and Saint-Denis on November 13, 2015, prehospital medical teams were confronted with a new and complex situation: support many victims of gunshot wounds, at multiple sites, in a context of insecurity. The implementation of the tactical concept of the Alpha Red Plan (extraction, triage, damage control, regulation and rapid evacuation) and the quality of relation between the emergency services (joint exercises, good communication) enabled to better manage this crisis.
\end{abstract}

Keywords Multiple victims $\cdot$ Triage $\cdot$ Prehospital medical care $\cdot$ Prehospital damage control

\section{Introduction}

Les attentats du 13 novembre 2015 ont confronté les équipes médicales préhospitalières à des difficultés spécifiques que sont l'engagement des équipes puis la médicalisation en

B. Frattini $(\bowtie) \cdot$ S. Boizat $\cdot$ S. Travers $\cdot$ M. Bignand

J.-P. Tourtier

1, place Jules Renard, F-75017 Paris

e-mail : benoit.frattini@pompiersparis.fr zone peu sécurisée et l'évacuation rapide de très nombreux blessés hémorragiques en zone urbaine.

\section{Engagement des équipes médicales}

L'enjeu pour les équipes médicales préhospitalières était de pouvoir disposer en temps réel des informations nécessaires pour permettre un engagement raisonné conciliant efficacité au service des victimes et exposition maîtrisée du personnel soignant. En situation de fusillades multiples, l'incertitude initiale (nombre et position des tireurs ? zones à risque ? présence d'explosifs ? axes dangereux ?) est une réalité fréquemment décrite [1]. Les réflexions en cours depuis plusieurs mois soulignaient l'importance du renseignement et du partage de l'information dès la prise d'appel et tout au long de l'intervention. L'objectif majeur était de pouvoir guider efficacement les équipes préhospitalières vers une zone de déploiement initiale puis des axes d'approche et d'évacuation sécurisés, tout en évitant, comme cela a souvent été le cas, d'interdire des zones qui a posteriori ne présentaient pas de danger. Les éléments permettant d'atteindre ces objectifs lors d'événements terroristes sont le recueil et la centralisation de l'information au centre de réception des appels par des personnels spécifiquement dédiés à ces missions de renseignement et la présence au sein de l'état-major opérationnel de responsables de bon niveau issus de chaque service (police, pompiers, Samu, associatifs). Les axes évidents d'amélioration seraient la création ou le renforcement de salles de crises communes entre les différents services préhospitaliers, le développement d'outils permettant de géolocaliser en interservices l'ensemble des engins, l'amélioration du partage d'informations entre services de secours, la meilleure identification sur le terrain des responsables des forces de Police et le développement de moyens de communication en propre en cas de défaillance ou d'arrêt volontaire des moyens de communication téléphoniques. 


\section{Médicalisation en zone peu sécurisée}

Plusieurs situations ont été rencontrées par les équipes médicales préhospitalières. Sur certains sites, le contexte tactique et médical était finalement proche de situations ayant conduit en milieu militaire au développement du Tactical Combat Casualty Care et du sauvetage au combat [2,3]. Certains enseignements issus des référentiels du service de santé des Armées se sont alors avérés très utiles aux équipes de terrain. Les « soins sous le feu » (care under fire) réalisés par les médecins des forces d'interventions étaient comme au combat centrés sur la mise en sécurité et la pose réflexe de garrots. Les « soins tactiques » (tactical care) réalisés par les équipes secouristes puis médicales dès mise à l'abri du blessé comprenaient dans l'ordre l'arrêt des hémorragies (garrots, pansements compressifs, packing hémostatiques...), la gestion des voies aériennes puis des traumatismes thoraciques (exsufflation, thoracostomie...), une stratégie de remplissage à faible volume (objectif : blessé conscient avec pouls radial perçu), l'administration d'acide tranéxamique, la prévention de l'hypothermie, et surtout l'évacuation rapide. La suite des soins était effectuée en cours d'évacuation (evacuation care) vers les services hospitaliers, les équipes étant accompagnées par des motards de la police sous forme de convois de plusieurs véhicules (Fig. 1). Dans un contexte particulièrement difficile, la préparation des équipes aux stratégies de damage control préhospitalier et l'utilisation de protocoles simples et partagés se sont une nouvelle fois avérées indispensables pour prioriser les actions utiles à la survie du patient et ne pas retarder les évacuations $[4,5]$.

Au sein de la Brigade de sapeurs-pompiers de Paris (BSPP), une formation médicale spécifique existe, ainsi que du matériel adapté et regroupé sous forme d'une trousse de damage control (Fig. 2). De plus, Samu et BSPP s'entrâ̂nent de concert. Du reste, le matin même des attentats, un exercice en commun avait lieu, en association avec des centres d'accueil de traumatisés sur le thème de fusillade sur lieux multiples.

\section{Évacuation rapide de très nombreux blessés hémorragiques}

En présence de victimes hémorragiques, et a fortiori lorsque le risque de sur-attentat ne peut pas être écarté, l'évacuation rapide des blessés est une priorité évidente. La stratégie élaborée par les services de secours parisiens au décours des attentats de Londres et Madrid, formalisée depuis 2006 dans le «plan rouge alpha », comprend schématiquement un ramassage, un triage médicalisé et une évacuation rapide des victimes vers les hôpitaux les plus adaptés à leur pathologie (le rôle de la régulation du Samu est majeur). Cette stratégie s'est avérée particulièrement adaptée aux attentats du 13 novembre 2015 et a permis une évacuation très rapide des victimes sur plusieurs sites. Après triage médical, le choix (également décrit dans le plan rouge alpha) de séparer parmi les patients classés urgences absolues (UA) ceux nécessitant une évacuation médicalisée (EU) et ceux pouvant, en l'absence d'un nombre suffisant de vecteurs médicalisés, être confiés à des véhicules secouristes après éventuel geste médical (U1) (avec accompagnement parfois par un médecin non urgentiste de circonstance) s'est également avéré pragmatique et pertinent pour de nombreux patients. Au total, 94 UA et 260 urgences relatives (UR) ont été prises en charge.

À l'inverse, les deux facteurs pouvant ralentir la manœuvre d'évacuation sont le délai d'arrivée d'un nombre suffisant de véhicules d'évacuation et l'attente de destination hospitalière. La régulation par le Samu, dans un contexte aussi complexe, a été pleinement efficace. Concernant

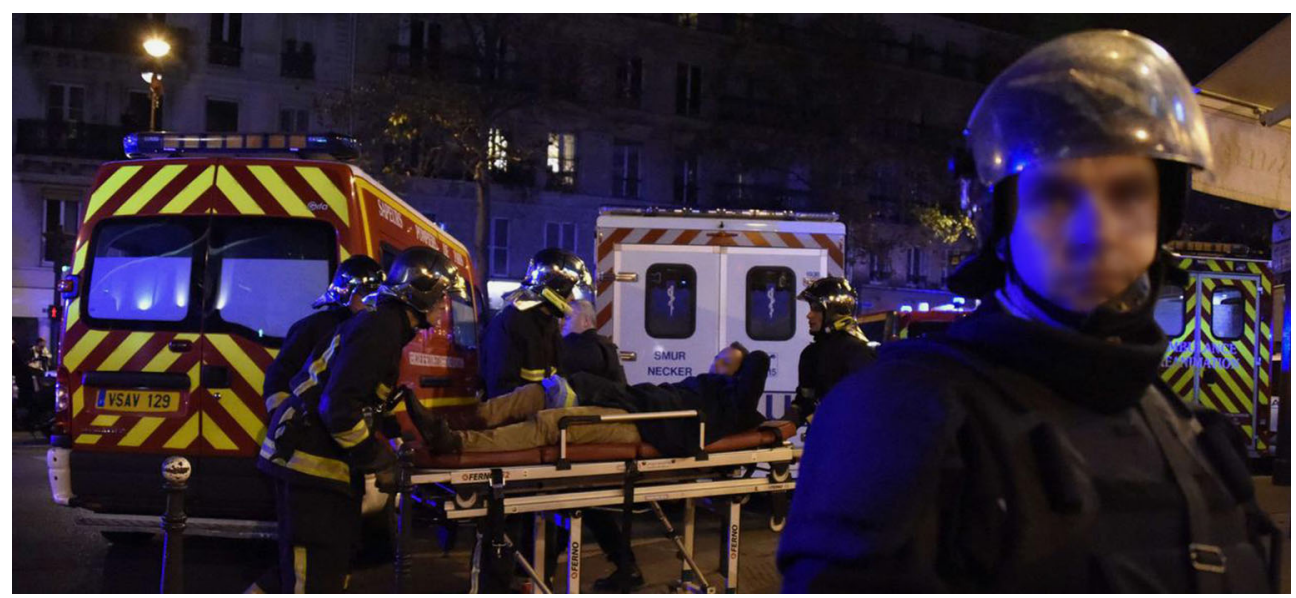

Fig. 1 Site du Bataclan : moyens médicaux de la Brigade de sapeurs-pompiers de Paris et du Samu devant le poste médical avancé « Brasserie la Royale » sous protection des forces de l'ordre 


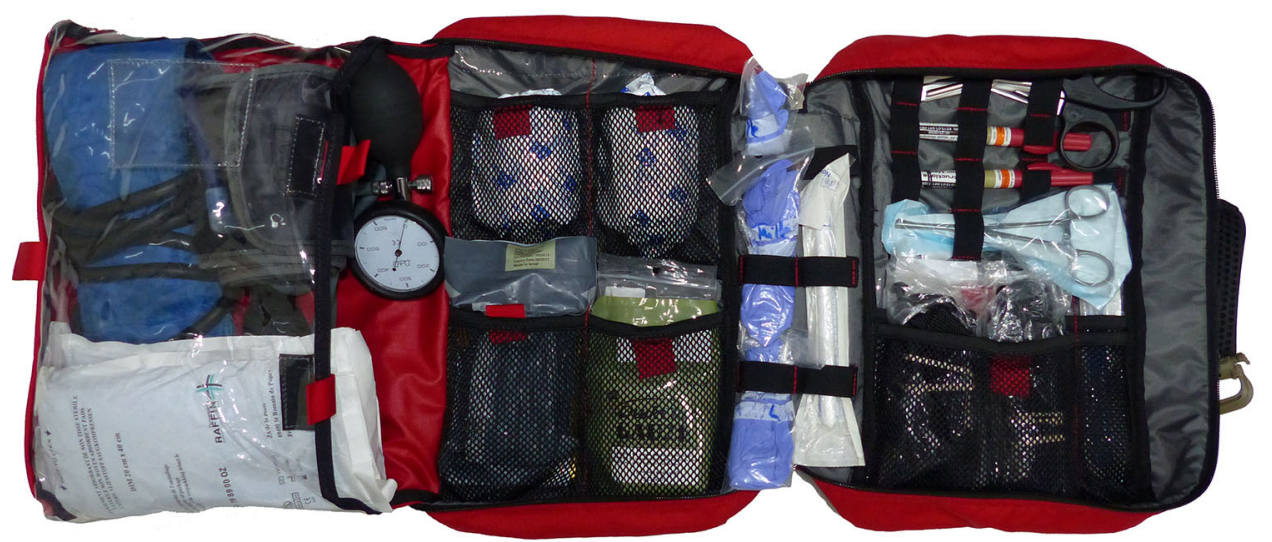

Fig. 2 Trousse « damage control» en dotation dans les ambulances de réanimation de la Brigade de sapeurs-pompiers de Paris. À gauche : garrots pneumatiques, pansements américains. Au milieu : bandes, pansements compressifs d'urgence, pansement hémostatique type QuikClot ${ }^{\circledR}$ Combat Gauze. À droite : garrots tourniquets, aiguilles d'exsufflation, bistouri à lame froide, pince de Kocher

l'accès rapide d'un nombre suffisant d'engins d'évacuation sur chaque site, les réflexions en cours portent sur une meilleure coopération interservice permettant de non seulement géolocaliser l'ensemble des engins mais aussi et surtout de mieux concilier manœuvres de police et accès des véhicules de secours. L'enjeu majeur que représente l'attribution des destinations hospitalières aux équipes de terrain incite par ailleurs à poursuivre les réflexions en cours sur la régulation médicale en situation de catastrophe (gestion en flux plutôt qu'en nombres de places, anticipation dès l'appel de filières d'évacuation...). Une voie spécifique d'amélioration semble encore la communication entre services pour que chaque équipe de terrain puisse être informée de sa destination en temps réel et quelles que soient les circonstances.

En conclusion, trois éléments ont participé à sauver des vies le 13 novembre 2015 : d'une part l'anticipation à travers des formations et des exercices communs BSPP-Samu, d'autre part la remarquable coopération sur le terrain des équipes médicales des pompiers et des Samu, enfin une synchronisation efficace des forces engagées par les directeurs de secours médicaux.
Liens d'intérêts : Les auteurs déclarent ne pas avoir de lien d'intérêt.

\section{Références}

1. Lockey DJ (2012) The shootings in Oslo and Utøya island July 22, 2011: Lessons for the International EMS community. Scand J Trauma Resusc Emerg Med 20:4

2. Butler FK, Smith DJ, Carmona RH (2015) Implementing and preserving the advances in combat casualty care from Iraq and Afghanistan throughout the US Military. J Trauma Acute Care Surg 79:321-6

3. Eastridge BJ, Mabry RL, Seguin P, et al (2012) Death on the battlefield (2001-2011): implications for the future of combat casualty care. J Trauma Acute Care Surg 73:431-7

4. Tourtier JP, Pelloux P, Dang Minh P, et al (2015) Charlie Hebdo attacks: lessons from the military milieu. Am J Emerg Med 33: 843

5. Tourtier JP, Palmier B, Tazarourte K, et al (2013) Le concept de damage control: extension préhospitalière du paradigme. Ann $\mathrm{Fr}$ Anesth Reanim 32:520-6 